Bull. Austral. Math. Soc.

$20 \mathrm{~K} 15,20 \mathrm{~K} 40$

VoL. 50 (1994) [177-195]

\title{
TORSION-FREE ABELIAN GROUPS TORSION OVER THEIR ENDOMORPHISM RINGS
}

\author{
Theodore G. Faticoni
}

\begin{abstract}
We use a variation on a construction due to Corner 1965 to construct (Abelian) groups $A$ that are torsion as modules over the ring End $(A)$ of group endomorphisms of $A$. Some applications include the failure of the Baer-Kaplansky Theorem for $\mathrm{Z}[X]$. There is a countable reduced torsion-free group $A$ such that $I A=A$ for each maximal ideal $I$ in the countable commutative Noetherian integral domain, End $(A)$. Also, there is a countable integral domain $R$ and a countable $R$-module $A$ such that (1) $R=\operatorname{End}(A),(2) T_{0} \otimes_{R} A \neq 0$ for each nonzero finitely generated (respectively finitely presented) $R$-module $T_{0}$, but (3) $T \otimes_{R} A=0$ for some nonzero (respectively nonzero finitely generated) $R$-module $T$.
\end{abstract}

\section{INTRODUCTION}

All groups considered in this paper are Abelian. The construction of groups with prescribed endomorphism ring originates with Corner's work [6] where he shows that each countable reduced torsion-free ring $R$ is the ring of group endomorphisms of a torsion-free group $A$. Inspection of the proof reveals that the group $A$ is an extension of $R$ by a free $Q R$-module. In [7], the free module is replaced by a direct sum of cyclic modules that are discrete in a complete Hausdorff linear topology on $R$. Other realisation Theorems show that each cotorsion-free ring $R$ is the endomorphism ring of a torsion-free group $A,[8]$, and again $A$ is an extension of a free $R$-module by a free $\mathbf{Q} R$-module. These constructions do not allow us to vary some of the subtler End $(A)$-module structure of $A$.

In [14, Theorem 3.1] we prove that if $R$ is a torsion-free ring of finite rank and if $M$ is a left $R$-module whose additive structure is a torsion-free group of finite rank then under mild hypotheses on $M$ there is an exact sequence

$$
0 \rightarrow M \longrightarrow A \longrightarrow \mathbf{Q} C \longrightarrow 0
$$

such that $C$ is a doubley generated free left $R$-module and $R=\operatorname{End}(A)$. While this construction allows us some flexibility in the $\operatorname{End}(A)$-structure of $A$, it still depends upon the existence of a copy of $R$ in $M$.

Received 31 May 1993

Copyright Clearance Centre, Inc. Serial-fee code: 0004-9729/94 \$A2.00+0.00. 
To describe our results, we require some terminology. If $M$ is a left $R$-module whose additive structure is a countable reduced torsion-free group then $M$ is called a Corner $R$-module. Given the left $R$-module $M$ such that $\operatorname{ann}_{R}(M)=0$, let $\Gamma(\mathbf{Q} M)$ be the set of annhilators of finite subsets of $\mathbf{Q} M$. Then $\Gamma(\mathbf{Q} M)$ generates a Hausdorff linear topology on $\mathbf{Q}$. Let $\widehat{\mathbf{Q R}}$ denote the completion of $\mathbf{Q} R$ under this topology, and observe that $\mathbf{Q M}$ is a left $\widehat{\mathbf{Q R}}$-module. Finally, let

$$
\widehat{\mathcal{O}}(M)=\{q \in \widehat{\mathbf{Q R}} \mid \boldsymbol{q} M \subset M\}
$$

Then $M$ is a left $\widehat{\mathcal{O}}(M)$-module, and we let $\widehat{\Gamma}(M)$ denote the set of annihilators of finite subsets of $M$ in $\widehat{\mathcal{O}}(M)$. Our main result is

Theorem 1.2. Let $R$ be a ring and let $M$ be a Corner $R$-module such that $\operatorname{ann}_{F}(M)=0$. There is an exact sequence (1.1) of left $R$-modules such that

1. $C$ is a direct sum of cyclic $R$-submodules of $M^{\left(\aleph_{0}\right)}$, the direct sum of countably many copies of $M$;

2. There is a topological isomorphism $\widehat{\mathcal{O}}(M) \cong \operatorname{End}(A)$ where $\widehat{\mathcal{O}}(M)$ is endowed with the topology generated by $\widehat{\Gamma}(M)$ and where $\operatorname{End}(A)$ is endowed with the finite topology.

When $\Gamma(\mathbf{Q} M)$ is discrete, (that is when 0 is the annihilator of a finite subset of $\mathbf{Q} M$ ), then $A$ is a self-small group and the construction is a generalisation of [14], which borrowed heavily from [15]. The present constructions will clone the elegant technique used in [7] to construct the short exact sequence (1.1).

Specific constructions contrast the theory of Abelian groups with the theory of modules over arbitrary integral domains. For example, let $R=\mathbf{Z}[X]$ and let $L$ be a torsion (as a $\mathrm{Z}[X]$-module) Corner $R$-module. Then $L$ is the torsion $R$-submodule of a (strongly) indecomposable mixed Corner $R$-module $A$. ( $A$ is mixed if $A$ is not a torsion module and $A$ contains a nonzero torsion $R$-submodule.) This is in contrast to the fact that no mixed group is indecomposable. Furthermore, each $X$-torsion Corner module $L$ imbeds in a (strongly) indecomposable $X$-torsion Corner module. As a consequence of these examples, the Baer-Kaplansky Theorem is false for mixed $Z[X]$-modules and for torsion $\mathbf{Z}[\boldsymbol{X}]$-modules.

Our constructions also illustrate the limits of some results from the literature. For example, it is well known that if $T$ is a right $R$-module and if $T_{0} \otimes_{R} A=0$ for each finitely generated $R$-submodule $T_{0} \subset T$ then $T \otimes_{R} A=0$. In 3.16 and 3.17 we construct a countable local commutative integral domain $R$ and a countable $R$-module $A$ such that (1) $R=$ End (A), (2) $T_{0} \otimes_{R} A \neq 0$ for each nonzero finitely generated (respectively finitely presented) $R$-module $T_{0}$, and (3) $T \otimes_{R} A=0$ for some nonzero (respectively nonzero finitely generated) $R$-module $T$. 
Lastly, if $A$ is a reduced torsion-free group of finite rank and if $\operatorname{End}(A)$ is either local or commutative then $I A \neq A$ for each proper right ideal $I \subset \operatorname{End}(A)$. However, we construct a reduced self-small torsion-free group $A$ such that $I A=A$ for each maximal ideal $I \subset \operatorname{End}(A)$ and such that $\operatorname{End}(A)$ is a local commutative Noetherian integral domain.

Our notation and terminology follow [3] and [15], and information on the finite topology can be found in [15]. As usual, $Z$ is the ring of rational integers, $Q$ is the field of rational numbers, and given a torsion-free group $G, \mathbf{Q} G$ is the divisible hull of $G$. We identify $\mathbf{Q}=\mathbf{Q} \otimes_{\mathbf{z}} G$. The notations Hom (and End) without subscripts denote the group (ring) of group homomorphisms. Also $\operatorname{ann}_{R}(X)=\{r \in$ $R \mid r x=0$ for each $x \in X\}$. See [1] for the elementary properties of ann $n_{R}$. We mention that if $x=\sum_{i} x_{i}$ is an independent sum, (that is if $r x=0$ implies $r x_{i}=0$ for each $i)$, then $\operatorname{ann}_{R}(x)=\operatorname{ann}_{R}\left(\left\{x_{i} \mid i\right\}\right)$. Also, because $M$ is a torsion-free group $\operatorname{ann}_{R}(k F)=\operatorname{ann}_{R}(F)=\bigcap_{x \in F} \operatorname{ann}_{R}(x)$ for each $0 \neq k \in \mathbb{Z}$ and subset $F \subset M$.

\section{Corner's CONSTRUCtion}

Throughout this paper, $R$ denotes an associative ring with identity, the term module means left $R$-module, and $M$ denotes a Corner module, (that is $M^{+}$is a countable reduced torsion-free Abelian group), such that $\operatorname{ann}_{R}(M)=0$. Similarly define Corner ring and Corner group.

Relativised finite topologies. Given a ring $S$ and a left $S$-module $L$

$$
\Gamma(S, L)=\Gamma(L)=\left\{\operatorname{ann}_{S}(F) \mid F \subset L \text { is finite }\right\}
$$

is the base of open neighbourhoods of zero for a linear topology on $S$ called the $L$ topology on $S$. We shall identify the $L$-topology on $S$ with $\Gamma(L)$. Observe that $\Gamma(L)$ is Hausdorff if and only if $\operatorname{ann}_{S}(L)=0$, and $\Gamma(L)$ is discrete if and only if $0 \in \Gamma(L)$ if and only if there is a finite set $F \subset \Gamma(L)$ such that $\operatorname{ann}_{S}(\dot{F})=0$.

Given a set $\Gamma$ of right ideals of $S$, the left $S$-module $N$ is called $\Gamma$-torsion if $N$ is a homomorphic image of a direct sum of cyclic modules of the form $S / I$ for some $I \in \Gamma$. Clearly $L$ is a $\Gamma(L)$-torsion left $S$-module.

If $\Gamma(L)$ is Hausdorff then we let $\widehat{S}_{L}$ denote the completion of $S$ in $\Gamma(L)$. Furthermore, $L$ is a left $\widehat{S}_{L}$-module as follows:

2.1. Let $\widehat{r} \in \widehat{S}_{L}$ and $x \in L$. There is a Cauchy net $\left\{r_{F}\right\} \subset S$, (indexed by the finite subsets of $L$ ), that converges to $\widehat{r}$. Choose a finite set $F \subset L$ such that $x \in F$ and $r_{E}-r_{F} \in \operatorname{ann}_{S}(x)$ for each finite set $F \subset E \subset L$. Then $\left(r_{F}-r_{E}\right) x=0$ so that $r_{F} x=r_{E} x$. Define $\widehat{r} \boldsymbol{x}=r_{F} x$. This makes $L$ a unital left $\widehat{S}$-module. Observe that $\operatorname{ann}_{\widehat{S}}(L)=0$. 
From the above discussion we have a linear topology $\Gamma(\mathbf{Q} M)=\Gamma(\mathbf{Q} R, \mathbf{Q} M)$ on $\mathbf{Q} R$ called the $\mathbf{Q} M$-topology. Because $\Gamma(\mathbf{Q} M)$ is Hausdorf and because $\mathbf{Q} R$ is a dense subring of the completion $\widehat{Q R}{ }_{\mathrm{QM}}=\widehat{\mathrm{QR}}$, ann $\widehat{\widehat{Q R}}(\mathbf{Q} M)=0$.

Let

$$
\widehat{\mathcal{O}}(M)=\operatorname{End}(M) \cap \widehat{Q R}=\{q \in \widehat{Q R} \mid q M \subset M\} .
$$

Then $M$ is left $\widehat{\mathcal{O}}(M)$-module, and $\operatorname{ann}_{\widehat{\mathcal{O}}(M)}(M)=0$. The $M$-topology on $\widehat{\mathcal{O}}(M)$ is generated by $\widehat{\Gamma}(M)=\Gamma(\widehat{\mathcal{O}}(M), M)$. Observe that

$$
\operatorname{ann}_{\widehat{\mathcal{O}}(M)}(F)=\operatorname{ann}_{\widehat{Q R}}(F) \cap \widehat{\mathcal{O}}(M)
$$

for each subset $F \subset \mathbf{Q} M$. Thus, $\widehat{\Gamma}(M)$ is the relativised topology in $\widehat{\mathcal{O}}(M)$ as a subring of $\widehat{\mathbf{Q R}}$. We shall use this last observation without fanfare.

LEMMA 2.2. $\widehat{\mathcal{O}}(M)$ is complete in the $M$-topology.

Proof: By the observations preceeding the Lemma, a Cauchy net $\left\{r_{F}\right\} \subset \widehat{\mathcal{O}}(M)$ is also a Cauchy net in $\widehat{Q R}$, so $\left\{r_{F}\right\}$ converges to an element $\widehat{r} \in \widehat{Q R}$, which clearly satisfies $\widehat{r} M \subset M$.

The CONSTRUCTION of $A$. To construct the exact sequence (1.1) we clone the process used in $[7$, p.66]. Unlike the proofs in $[14, p .10]$ and $[15, p .233]$, the process in [7] does not require a local/global argument.

There is an uncountable domain $\mathbf{P} \subset \widehat{Z}$ such that each element of $\mathbf{P}$ is a rational multiple of a unit in $\mathbf{P},[6$, Section 2], and there is a countable subring $I I \subset \mathbf{P}$ such that the following Lemma is true.

Lemma 2.3. Let $\mathcal{L} \subset \mathbf{P}$ be a set that is linearly independent over $\Pi$, and let $\left\{x_{\lambda} \mid \lambda \in \mathcal{L}\right\} \subset \mathbf{Q} M$.

1. If $\sum_{\lambda \in \mathcal{L}} \lambda x_{\lambda}=0$ then $x_{\lambda}=0$ for each $\lambda \in \mathcal{L}$.

2. $\operatorname{ann}_{R}\left(\sum_{\lambda \in \mathcal{L}} \lambda x_{\lambda}\right)=\operatorname{ann}_{R}\left(\left\{x_{\lambda} \mid \lambda \in \mathcal{L}\right\}\right)$.

The next Lemma constructs a direct sum of cyclic $\Gamma(M)$-discrete modules.

LEMma 2.4. There is a Corner submodule $N \subset \widehat{M}$ such that

1. $M \cap N=0$;

2. For each finite set $E \subset M$ there is $u_{E} \in N$ such that $\operatorname{ann}_{R}\left(u_{E}\right)=$ $\operatorname{ann}_{R}(E)$;

3. $\quad N$ is a direct sum of cyclic submodules of $M^{\left(\aleph_{0}\right)}$. 
Proof: Because $M$ is countable the set $\mathcal{P}_{0}(M)$ of finite subsets of $M$ is countable, and because $P$ is uncountable, there is a countable set

$$
\mathcal{L}=\left\{1, \lambda_{E_{x}} \mid E \in \mathcal{P}_{0}(M) \text { and } x \in E\right\} \subset P
$$

that is linearly independent over II. Given $E \in \mathcal{P}_{0}(M)$ let

$$
u_{E}=\sum_{x \in E} \lambda_{E x} x
$$

and let

$$
N=\sum_{E \in \mathcal{P}_{0}(M)} R u_{E}
$$

Because $\mathcal{L}$ is linearly independent over II 2.3.1 implies that the sum $\sum_{E \in \mathcal{P}_{0}(M)} R u_{E}$ is direct, and that $M \cap N=0$. Thus $N$ satisfies 2.4.1.

Fix $E \in \mathcal{P}_{0}(M)$, and consider the element $u_{E} \in N$ given in 2.5. Because $\mathcal{L}$ is linearly independent over $I I, \operatorname{ann}_{R}\left(u_{E}\right)=\operatorname{ann}_{R}(E), 2.3 .2$, so that $N$ satisfies 2.4.2.

Finally, given $E \in \mathcal{P}_{0}(M), 2.4 .2$ shows that there is an imbedding $R u_{E} \rightarrow M^{(E)}$ such that $u_{E} \mapsto \bigoplus_{x \in E} x$. Then $N$ can be imbedded in $M^{\left(K_{0}\right)}$ as required by 2.4.3.

Throughout the sequel, $\boldsymbol{N}$ denotes the module constructed in 2.4 .

2.6. In as much as $M \otimes N$ is countable there exists a set of units

$$
\mathcal{A}=\left\{\varepsilon_{m n} \mid m \in M, n \in N\right\} \subset \mathbf{P}
$$

that is algebraically independent over $\Pi$. Let $A$ be the pure subgroup of $\widehat{M}$ generated by $M, N$, and the $R \varepsilon_{m n}$,

$$
\begin{aligned}
A & =\left\langle M, N, R(m \oplus n) \epsilon_{m n} \mid m \in M, n \in N\right\rangle_{*} \\
& =\widehat{M} \cap \mathbf{Q}\left(M+N+\sum_{m \in M, n \in N} R(m \oplus n) \epsilon_{m n}\right)
\end{aligned}
$$

Observe that $A$ is a Corner submodule of $\widehat{M}$.

Proposition 2.7. Let $\epsilon_{m n}$ be as in 2.6.

1. $\sum_{m \in M, n \in N} R(m \oplus n) \epsilon_{m n}$ is a direct sum of cyclic submodules of $M^{\left(\aleph_{0}\right)}$.

2. $A$ is a left $\widehat{\mathcal{O}}(M)$-module. 
Proof: (1) The independence of the sum follows from 2.3.1. Because $\epsilon_{m n}$ is a unit in $\mathbf{P}, R(m \oplus n) \epsilon_{m n} \cong R(m \oplus n) \subset M \oplus N$, and by 2.4 .3 there is an imbedding $M \oplus N \rightarrow M \oplus M^{\left(\kappa_{0}\right)} \cong M^{\left(\kappa_{0}\right)}$.

(2) Observe that $\mathbf{Q} A$ is a left $\mathbf{Q} R$-module, so by $2.1 \mathbf{Q} A$ is a left $\widehat{\mathbf{Q R}}$-module. Because $M$ is left $\widehat{\mathcal{O}}(M)$-module, $M$ is a left $\widehat{\mathcal{O}}(M)$-module, and hence $\widehat{M} \cap \mathbf{Q} A=A$ is a left $\widehat{\mathcal{O}}(M)$-module.

ENDOMORPHISMS OF $A$. The proof of the next Lemma is close to that in $[7, \mathrm{p} .67]$, so the process of comparing coefficients has been left to the reader.

Lemma 2.8. Let $\eta \in \operatorname{End}(A)$. For each $m \in M, n \in N$ there is an $r_{m n} \in \mathbf{Q} R$ such that $\eta(m)=r_{m n} m$ and $\eta(n)=r_{m n} n$.

Proof: Let $\eta \in \operatorname{End}(A)$. Because $A$ is a pure subgroup of $\widehat{M}, \eta$ lifts to a $\widehat{Z}$ module homomorphism $\eta: \widehat{M} \rightarrow \widehat{M}$, and because $A$ is torsion-free $\eta$ lifts to a $\mathbf{Q}$-vector space homomorphism $\eta: \mathbf{Q} A \rightarrow \mathbf{Q} A$.

Let $m \in M, n \in N$. By 2.6 there is a finite subset $E \subset M \oplus N$ such that

$$
\begin{aligned}
\eta\left((m \oplus n) \epsilon_{m n}\right) & =\eta(m \oplus n) \epsilon_{m n} \\
& =x+\sum_{m^{\prime} \oplus n^{\prime} \in E} r_{m^{\prime} n^{\prime}}\left(m^{\prime} \oplus n^{\prime}\right) \epsilon_{m^{\prime} n^{\prime}} \\
\eta(m \oplus n) & =y+\sum_{m^{\prime} \oplus n^{\prime} \in E} s_{m^{\prime} n^{\prime}}\left(m^{\prime} \oplus n^{\prime}\right) \epsilon_{m^{\prime} n^{\prime}}
\end{aligned}
$$

where $x, y \in \mathbf{Q}(M \oplus N)$ and $r_{m^{\prime} n^{\prime}}, s_{m^{\prime} n^{\prime}} \in \mathbf{Q} R$. Assume without loss of generality that $m \oplus n \in E$. As in [7], substitute (2) and (3) into (1) and then use 2.3.1 to compare coefficients in (1) and (2) to prove that $x=r_{m^{\prime} n^{\prime}}\left(m^{\prime} \oplus n^{\prime}\right)=0$ for each $m^{\prime} \oplus n^{\prime} \in E$, $s_{m^{\prime} m^{\prime}}\left(m^{\prime} \oplus n^{\prime}\right)=0$ for each $m \oplus n \neq m^{\prime} \oplus n^{\prime} \in E$, and $r_{m n}(m \oplus n)=y=\eta(m \oplus n)$.

Finally, because $m \oplus 0,0 \oplus n \in M \oplus N$ the above argument produces $r_{m}, r_{n} \in R$ such that $\eta(m)=r_{m} m, \eta(n)=r_{n} n$, and $\eta(m \oplus n)=r_{m n}(m \oplus n)=r_{m} m \oplus r_{n} n$. Because $M \oplus N$ is a direct sum of modules, $r_{m n} m=r_{m} m$ and $r_{m n} n=r_{n} n$. This completes the proof.

The rest of the proof is different enough from $[7$, p.67-68] that we give a more detailed account. However, the idea is the same: realise a given endomorphism $\eta: A \rightarrow$ $A$ as left multiplication by the limit of a Cauchy net (sequence) in $R$.

LEMMA 2.9. There is an isomorphism of rings $\widehat{\mathcal{O}}(M) \cong \operatorname{End}(A)$.

ProOf: By 2.7.2 $A$ is an $\widehat{\mathcal{O}}(M)$-submodule of $\widehat{M}$. Inasmuch as $r A=0$ implies $r \widehat{M}=0$ implies $r=0$ for $r \in \widehat{\mathcal{O}}(M)$, there is an imbedding of rings $\widehat{\mathcal{O}}(M) \rightarrow \operatorname{End}(A)$. We claim this imbedding is an isomorphism. 
Let $\eta \in \operatorname{End}(A)$ and let $E \subset M$ be finite. By 2.4.2 there is an element $u_{E} \in N$ such that $\operatorname{ann}_{R}\left(u_{E}\right)=\operatorname{ann}_{R}(E)$.

Next, 2.8 states that for each $m \in E$ there exists $r_{m E} \in \mathbf{Q} R$ such that $\eta(m)=$ $r_{m E} m$ and $\eta\left(u_{E}\right)=r_{m E} u_{E}$. Let $r_{E}$ be any one of the $r_{m E}$. Then $r_{m E} u_{E}=\eta\left(u_{E}\right)=$ $r_{E} u_{E}$ for each $m \in E$, so that

$$
r_{E}-r_{m E} \in \operatorname{ann}_{R}\left(u_{E}\right)=\operatorname{ann}_{R}(E) \subset \operatorname{ann}_{R}(m) .
$$

Then $\left(r_{E}-r_{m E}\right) m=0$ shows that $r_{E} m=r_{m E} m=\eta(m)$ for each $m \in E$.

Subsequently, the set $\left\{r_{E} \mid E \subset M\right.$ is finite $\}$ is a Cauchy net in $\widehat{Q R}$ under the $\mathbf{Q} M$-topology. Let $\widehat{r}$ denote the limit in $\widehat{Q R}$ of this Cauchy net. By $2.1 \widehat{r} m=r_{E} m=$ $\eta(m)$ for each finite set $E \subset \mathbf{Q} M$ and each $m \in E$. Thus $[\eta-\widehat{r}](M)=0$. Inasmuch as $A \subset \widehat{M}$ is a reduced group, we can proceed as usual (see for example $[6,7,8,13$, $14,15])$, to show that $\eta=\widehat{r} \in \widehat{\mathcal{O}}(M)$. Therefore, the imbedding $\widehat{\mathcal{O}}(M) \rightarrow \operatorname{End}(A)$ is an isomorphism.

The proof of the next lemma is identical to that given in $[7, p .68-69]$, and is thus left to the reader.

LEMMA 2.10. The $M$-topology on $\widehat{\mathcal{O}}(M)$ is equivalent to the relativised finite topology on $\widehat{\mathcal{O}}(M)$ as a subring of $\operatorname{End}(A)$.

Proof of Theorem 1.2: Given the Corner module $M$ construct $N \subset \widehat{M}$ and $A$ as in 2.6. Let

$$
C=N \oplus \sum_{m \in M, n \in N} R(m \oplus n) \epsilon_{m n}
$$

Because $M \subset A \subset \widehat{M}, A / M$ is a torsion-free divisible group, and thus $A / M \cong \mathbf{Q} C$ as modules. By 2.4.3 and 2.7.1 $C$ is a direct sum of cyclic submodules of $M^{\left(\aleph_{0}\right)}$. Thus 1.2.1 is satisfied.

Furthermore, by 2.9 and 2.10 there is a topological isomorphism $\widehat{\mathcal{O}}(M) \cong \operatorname{End}(A)$, where $\widehat{\mathcal{O}}(M)$ is endowed with the $M$-topology and where $\operatorname{End}(A)$ is endowed with the finite topology. This completes the proof of Theorem 1.2.

The proof of the Corollary follows immediately from 2.6 .

Corollary 2.11. Let $R$ be a ring, let $M$ be a Corner module such that $\operatorname{ann}_{R}(M)=0$, and let (1.1) be the sequence constructed by Theorem 1.2. Then the exact sequence of left modules

$$
\mathbf{0} \longrightarrow \mathbf{Q} M \longrightarrow \mathbf{Q} A \longrightarrow \mathbf{Q} C \longrightarrow 0
$$

formed by applying $\mathbf{Q} \otimes \mathbf{z} \cdot$ to (1.1) is split exact. 
Self-SMALl GROUPS. A variation on the above construction produces modules $A$ such that $\operatorname{End}(A)$ is discrete in the finite topology. We begin by constructing a unimodular element.

Lemma 2.12. Let $M$ be a Corner module. There is a $u \in \widehat{M}$ such that $\operatorname{ann}_{R}(u)=\operatorname{ann}_{R}(M)$.

Proof: Because $M$ is countable we can write $M=\left\{m_{i} \mid i=1,2, \ldots\right\}$. Choose a set $\left\{\lambda_{i} \mid i=1,2, \ldots\right\} \subset \mathbf{P}$ that is linearly independent over $\Pi$, (see the remarks preceeding 2.3). Then $u=\sum_{i=1}^{\infty} p^{i} \lambda_{i} m_{i}$ is a convergent sum in $\widehat{M}$. Let $u_{k}=\sum_{i=1}^{k} p^{i} \lambda_{i} m_{i}$.

Given $r \in \operatorname{ann}_{R}(u)$ and a positive $k \in \mathbf{Z}$ then $r u_{k}=r u_{k}-r u \in p^{k} \widehat{M}$. That is, the $p$-height of $r u_{k}$ is at least $k$. However, because the finite sum $\sum_{k=1}^{k} p^{i} \lambda_{i} m_{i}$ is independent, 2.3.1, the $p$-height of $r u_{k}$ is at most the $p$-height of $r\left(p \lambda_{1} m_{1}\right)$. We arrive at a contradiction unless $r u_{k}=0$. But then by 2.3.2

$$
r \in \bigcap_{k>0} \operatorname{ann}_{R}\left(m_{1}, \ldots, m_{k}\right)=\operatorname{ann}_{R}\left(m_{1}, m_{2}, \ldots\right)=\operatorname{ann}_{R}(M)=0
$$

as required by the Lemma.

A group $A$ is self-small if the natural imbedding $\operatorname{Hom}(A, A)^{(c)} \rightarrow \operatorname{Hom}\left(A, A^{(c)}\right)$ is an isomorphism for each cardinal $c$. Let

$$
\mathcal{O}(M)=\{q \in \mathbf{Q R} \mid q M \subset M\}
$$

The next Theorem extends [14, Theorem 3.1].

THEOREM 2.13. Let $R$ be a countable ring and let $M$ be a Corner module such that $\operatorname{ann}_{R}(M)=0$. There is an exact sequence (1.1) of modules such that

1. $C$ is a free module;

2. $\mathcal{O}(M) \cong \operatorname{End}(A)$; and

3. $A$ is self-small Corner group.

Proof: By 2.12 there exists a $u \in \widehat{M}$ such that ann $+_{R}(u)=\operatorname{ann}_{R}(M)=0$, and it follows from 2.3.1 that $M \cap R u=0$. Then $M \oplus R u \subset \widehat{M}$. Because $R$ and $M$ are countable, there is a set

$$
\mathcal{A}=\left\{\epsilon_{m} \mid m \in M\right\} \subset \mathbf{P}
$$

that is algebraically independent over II. Let

$$
C=R u+\sum_{m \in M} R(m \oplus u) \epsilon_{m}
$$


and let $A$ be the purification of $M$ and $C$ in $\widehat{M}, A=\langle M, C\rangle_{*}$. As in $2.6 A$ is a left $R$ submodule of $\widehat{M}$, and by 2.3.2 $R \cong R u \cong R(m \oplus u) \epsilon_{m}$. Furthermore, because $\mathcal{A} \cup\{1\}$ is linearly independent over $\Pi, 2.3 .1, C$ is a free module. Inasmuch as $M \subset A \subset \widehat{M}$, $A / M \cong \mathbf{Q} C$, and hence $A$ is the middle term of an exact sequence (1.1). Thus 2.13.1 is satisfied.

Because $A$ is a left $R$-module and because $\operatorname{ann}_{\mathcal{O}(M)}(A)=\operatorname{ann}_{\mathcal{O}(M)}(M)=0$ there is an imbedding $\mathcal{O}(M) \rightarrow \operatorname{End}(A)$ that sends each $r \in \mathcal{O}(M)$ to left multiplication by $r$. We claim that this imbedding is an isomorphism.

As in 2.9 one proves that for each $m \in M$ there is a scalar $r_{m} \in \mathbf{Q R}$ such that $\eta(m)=r_{m} m$ and $\eta(u)=r_{m} u$. Let $r_{0}$ be one of the $r_{m}$. Then for each $m \in M$, $r_{0} u=\eta(u)=r_{m} u$, so that $r_{0}-r_{m} \in \operatorname{ann}_{Q R}(u)=0$. Hence $r_{m}=r_{0}$ for each $m \in M$ and therefore $\eta(m)=r_{0} m$ for each $m \in M$. As in $2.9, \eta=r_{0} \in \operatorname{End}(A)$, and hence $R \cong \operatorname{End}(A)$.

Lastly, $A$ is a countable group because $M$ is countable, and because $u \in A$ the finite topology on $\operatorname{End}(A)$ is discrete. Then by [5, Proposition 2.1] $A$ is a self-small group. This completes the proof.

\section{Examples}

We use 1.2 and 2.13 to construct examples of modules with various properties.

ENDOMORPHISM RINGS OF FAITHFULLY FLAT MODULES. In [14, Corollary 3.10] it is shown that each finite rank Corner ring is the group endomorphism ring of a faithfully flat Corner module of finite rank. This is extended in [9] to include all cotorsion-free rings, but the cardinality of the faithfully flat Corner module is quite large. The next few results verify that such large cardinalities can be avoided when $R$ is a Corner ring.

Lemma 3.1. Let $R$ be a ring, let $M$ be a Corner module such that $\operatorname{ann}_{R}(M)=$ 0 , and let (1.1) be the exact sequence constructed in 1.2. Assume $C$ is a free left $R$-module.

1. Given a right $\operatorname{End}(A)$-module $N$ and if $N \otimes_{\operatorname{End}(A)} M \neq 0$ then $N \otimes_{\operatorname{End}(A)}$ $A \neq 0$.

2. If $\mathbf{Q} C$ is a flat $\hat{\mathcal{O}}(M)$-module then $A$ has flat dimension $\leqslant k$ as a left End $(A)$-module if and only if $M$ has flat dimension $\leqslant k$ as a left $\widehat{\mathcal{O}}(M)$ module.

Proof: (1) Let $\mathbf{E}=\operatorname{End}(A)$. If $N \otimes_{E} A=0$ then there is a short exact sequence

$$
\operatorname{Tor}_{E}^{1}(N, \mathbf{Q} C) \longrightarrow N \otimes_{E} M \longrightarrow N \otimes_{E} A=0 .
$$

Because $C$ is free, $\mathbf{Q} C$ is a flat left $E$-module, and so $\operatorname{Tor}_{E}^{1}(N, \mathbf{Q} C)=0$. Hence $N \otimes_{E} M=0$. This proves part 1 . 
(2) Recall from 1.2 that $\mathbf{E}=\widehat{\mathcal{O}}(M)$. Because $\mathbf{Q} C$ is a flat left $\widehat{\mathcal{O}}(M)$-module, an application of $X \otimes_{E} \cdot$ to (1.1) produces the long exact sequence

$$
\operatorname{Tor}_{\mathbf{E}}^{k+2}(X, \mathbf{Q} C) \longrightarrow \operatorname{Tor}_{\mathbf{E}}^{k+1}(X, M) \longrightarrow \operatorname{Tor}_{\mathbf{E}}^{k+1}(X, A) \longrightarrow \operatorname{Tor}_{\mathbf{E}}^{k+1}(X, \mathbf{Q} C)
$$

in which the first and last terms are 0 . Hence $A$ has flat dimension $\leqslant k$ as a left E-module if and only if $M$ has flat dimension $\leqslant k$ as a left $\widehat{\mathcal{O}}(M)$-module.

The next result extends [14, Corollary 3.10] to Corner groups.

Corollary 3.2. Let $R$ be a Corner ring. There is a self-small faithfully flat Corner group $A$ such that $R=\operatorname{End}(A)$.

PROOF: Let $M$ be a nonzero free left $R$-module on at most countably many generators to construct $A$ as in 2.13 , and then apply 3.1 .

DIMENSIONS OVER ENDOMORPHISM RINGS. Several papers on Abelian groups investigate the existence of left $R$-modules $A$ such that $R=\operatorname{End}(A)$ and such that $A$ has specified homological dimension. (See the references in [9].) The results in [9, Section 3] construct cotorsion-free groups of large cardinality with prespecified flat or projective dimension over their endomorphism ring. If the techniques from [9] are combined with our 1.2, then we can construct groups of small cardinality having prescribed dimension.

Theorem 3.3. Let $R$ be a Corner ring, and let $n=\operatorname{wgd}(R)$ denote the weak global dimension of $R$.

1. If $R$ possesses a left module of flat dimension $k+1$ then there is a selfsmall Corner group $A_{k}$ such that $R=\operatorname{End}\left(A_{k}\right)$ and such that $A_{k}$ has projective dimension $k$ over $R$.

2. If $n<\infty$ then for each $0 \leqslant k<n$ there is a self-small Corner group $A_{k}$ such that $R=\operatorname{End}\left(A_{k}\right)$ and such that $A_{k}$ has flat dimension $k$ over $R$.

3. If $n=\infty$ then there is a self-small Corner group $A_{\infty}$ such that $R=$ End $\left(A_{\infty}\right)$ and such that $A_{k}$ has flat dimension $\infty$ over $R$.

TheORem 3.4. Let $R$ be a Corner ring, and let $n=\operatorname{wgd}(R)$ denote the left global dimension of $R$.

1. If $R$ possesses a left module of projective dimension $k+1$ then there is a self-small Corner group $A_{k}$ such that $R=\operatorname{End}\left(A_{k}\right)$ and such that $A_{k}$ has projective dimension $k$ over $R$.

2. If $n<\infty$ then for each $1 \leqslant k<n$ there is self-small Corner group $A_{k}$ such that $R=\operatorname{End}\left(A_{k}\right)$ and such that $A_{k}$ has projective dimension $k$ over $R$.

3. If $n=\infty$ then there is a self-small Corner group $A_{\infty}$ such that $R=$ End $\left(A_{\infty}\right)$ and such that $A_{k}$ has projective dimension $\infty$ over $R$. 
ProOF: To prove either of the above Theorems, proceed exactly as in the proofs of [9, Theorems 3.1 and 3.8], but appeal to 2.13 instead of [9, Theorem 2.14]. The rest carries over exactly.

Commutative Endomorphism RINGS. Throughout the rest of this Section $R$ denotes a commutative ring, the term module means $R$-module, and $M$ denotes a Corner module that satisfies (i) and (ii) of 1.2. Moreover, $S$ denotes a Corner integral domain, and $X$ is indeterminant over $S$.

We list the following terminology for the sake of clarity.

The $S[X]$-module $L$ is an $X$-torsion $S[X]$-module if for each $x \in L$ there is an integer $k>0$ such that $X^{k} x=0, L$ is an $X$-divisible $S[X]$-module if $X L=L$, and $L$ is an $X$-reduced $S[X]$-module if $L$ does not contain a nonzero $X$-divisible $S[X]$ submodule.

The $S[X]$-module $L$ is a torsion $S[X]$-module if for each $x \in L$ there is a nonzero $p(X) \in S[X]$ such that $p(X) x=0$, and $L$ is a mixed $S[X]$-module if $L$ is not a torsion $S[X]$-module and $L$ has nonzero torsion $S[X]$-submodule.

Given $Y \subset R$ let $(Y)$ denote the ideal generated by $Y$, and let $\langle Y\rangle$ be the pure ideal of $R$ generated by $Y$. That is $\langle Y\rangle$ is the ideal of $R$ such that $\langle Y\rangle /(Y)$ is the torsion subgroup of $R /(Y)$.

TORSION SUBMODULES OF MIXED MODULES. In an abuse of terminology, we shall call $L$ a self-small module if $L$ is a module whose additive structure is a self-small Abelian group.

Proposition 3.5. If $L$ is a Torsion Corner $S[X]$-module then $L$ is the torsion $S[X]$-submodule of a self-small mixed Corner $S[X]$-module $A$ such that $S[X]=$ End $(A)$.

Proof: Let $R=S[X]$, (so that module refers to an $S[X]$-module.) Given the torsion Corner module $L$ notice that $M=R \oplus L$ is a Corner module such that $\operatorname{ann}_{R}(M)=0$. An application of 2.13 to $M$ yields a self-small Corner module $A$ such that $R=\operatorname{End}(A)$ and $A / M$ is a torsion-free module. (See 2.13.1.) Then $L$ is the torsion submodule of $A$.

EXAMPLE 3.6. There is a self-small mixed Corner module $A$ such that $Z[X]=$ End $(A)$ and each cyclic Corner $\mathrm{Z}[X]$-module imbeds as a $\mathrm{Z}[X]$-submodule of $A$.

Proof: Let $R=Z[X]$ and let

$$
M=\bigoplus\{R /\langle p(X)\rangle \mid p(X) \in R\}
$$

We claim that $M$ is a torsion Corner module. 
Clearly $M$ is a torsion module. Let $0 \neq I=\langle p(X)\rangle \subset R$. The usual argument using the Euclidean division algorithm shows that $R / I$ is generated as a group by the finitely many cosets $X^{k}+I$ where $k=0, \ldots, \operatorname{deg}(p(X))-1$. Because these cosets are linearly independent over $\mathbf{Z}, R / I$ is a finitely generated torsion-free group, and hence $M$ is a torsion Corner module, as claimed.

It is an easy matter to show that $\mathcal{O}(M)=R$ and that $\operatorname{ann}_{R}(M)=0$. Then use 3.5 to construct a self-small Corner module $A$ such that $M$ is the torsion submodule of $A$ and $R=\operatorname{End}(A)$. Observe from the proof of 3.5 that $R \subset A$. Also, by the definition of $M$ each cyclic torsion Corner module imbeds in $M \subset A$.

The next example gives an idea of the different types of endomorphism rings that are realised by Theorem 1.2 .

EXample 3.7. There is a torsion Corner $\mathrm{Z}[X]$-module $A$ such that $\operatorname{End}(A)=$ $\prod_{p(X)} \mathrm{Z}[X] /\langle p(X)\rangle$ where $p(X)$ ranges over the irreducible polynomials in $\mathrm{Z}[X]$.

Proof: Let $R=\mathbf{Z}[X]$ and let

$$
M=\oplus\{R /\langle p(X)\rangle \mid p(X) \in R \text { is irreducible }\} .
$$

As in 3.6 $M$ is a torsion Corner module such that $\operatorname{ann}_{R}(M)=0$.

Now, because $\mathbf{Q R}$ is a pid the Chinese Remainder Theorem can be applied to show that $Q R$ is a dense subring of $\operatorname{End}_{Q R}(Q M)$. Futhermore, it is evident that the $\mathbf{Q} M$-topology on $\mathbf{Q R}$ is equivalent to the relativised topology on $\mathbf{Q} R$ as a subring of $\operatorname{End}_{\mathbf{Q}}(\mathbf{Q} M)$. Because $\operatorname{End}_{\mathbf{Q}}(\mathbf{Q} M)$ is a complete Hausdorff space in finite topology we have that $\widehat{Q R} \cong \operatorname{End}_{\mathbf{Q} R}(\mathbf{Q} M)$.

Lastly, it can be shown that

$$
\operatorname{End}_{\mathbf{Q} R}(\mathbf{Q} M) \cong \prod\{\mathbf{Q} R /(p(X)) \mid p(X) \in \mathbf{Q} R \text { is irreducible }\},
$$

and thus that

$$
\widehat{\mathcal{O}}(M)=\prod\{R /\langle p(X)\rangle \mid p(X) \in R \text { is irreducible }\} .
$$

Thus, by 1.2 there is an torsion Corner module $A$ such that $\widehat{\mathcal{O}}(M)=\operatorname{End}(A)$.

The Baer-Kaplansky Theorem. We use the following two $X$-torsion Corner $S[X]$ modules to show that the Baer-Kaplansky Theorem is not true for $Z[X]$-modules.

3.8. $M=\oplus\left\{S[X] /\left(X^{k}\right) \mid k=1,2, \ldots\right\}$.

3.9. $M$ is the $S[X]$-module whose additive structure is a free $S$-module with generators $\left\{b_{1}, b_{2}, \ldots\right\}$, and whose relations as an $S[X]$-module are given by $X b_{k+1}=b_{k}$ for $k>1$ and $X b_{1}=0$.

The proof of the next Lemma is an exercise. 


\section{LEMMA 3.10 .}

1. The $S[X]$-module given in 3.8 is an $X$-reduced $X$-torsion Corner $S[X]$ module such that $\operatorname{ann}_{S[X]}(M)=0, \mathcal{O}(M)=S[X], \hat{\mathcal{O}}(M)=S[[X]]$, and $\widehat{\Gamma}(M)$ is the $X$-adic topology on $S[[X]]$.

2. The $S[X]$-module given in 3.9 is an $X$-divisible $X$-torsion Corner $S[X]$ module such that $\operatorname{ann}_{S[X]}(M)=0, \mathcal{O}(M)=S[X], \widehat{\mathcal{O}}(M)=S[[X]]$, and $\widehat{\Gamma}(M)$ is the $X$-adic topolgoy on $S[[X]]$.

EXAMPLE 3.11. Let $M$ be an $X$-torsion Corner $S[X]$-module such that $\mathcal{O}(M)=S[X]$. (For example, take $M$ to be the $S[X]$-module given in 3.8 or 3.9.) There is an $X$-torsion Corner $S[X]$-module $A$ such that $M \subset A, \operatorname{End}(A)=S[[X]]$, and $A / M$ is a divisible group.

Proof: Let $R=S[X]$ and observe that if $M$ is an $X$-torsion Corner $S[X]$-module such that $\operatorname{ann}_{R}(M)=0$ then the $M$-topology equals the $X$-adic topology on $S[X]$. Thus $\widehat{Q R}=(\mathbf{Q S})[[X]]$, the ring of power series over $\mathbf{Q S}$. It is readily shown using 2.1 that $\widehat{\mathcal{O}}(M)=S[[X]]$, so by 1.2 there is an $X$-torsion Corner $S[X]$-module $A$ such that $M \subset A, \operatorname{End}(A)=S[[X]]$, and $A / M$ is a divisible group.

The groups constructed in 3.11 are examples of strongly indecomposable countable groups with uncountable endomorphism rings. This refines a construction given in [13] in answer to a question of Reid, [16].

The Baer-Kaplansky Theorem states that if $A$ and $B$ are torsion groups and if $\operatorname{End}(A) \cong \operatorname{End}(B)$ as rings then $A \cong B$. The following shows that the BaerKaplansky Theorem is not true for torsion $\mathrm{Z}[X]$-modules. Recall that if $R=\operatorname{End}(A)$ is a commutative ring then $\operatorname{End}(A)=\operatorname{End}_{R}(A)$.

Proposition 3.12. There are $X$-torsion Corner $S[X]$-modules $A$ and $B$ such that $\operatorname{End}_{S[X]}(A) \cong \operatorname{End}_{S[X]}(B)$ as rings, but $\operatorname{Hom}_{S[X]}(B, A)=0$.

Proof: Let $R=S[X]$, let $K$ be the module given in 3.8, let $M$ be the module given in 3.9. By 3.11 there are $X$-torsion Corner modules $A$ and $B$ such that $K \subset A$, $M \subset B, \operatorname{End}(A)=\operatorname{End}(B)=S[[X]]=\widehat{R}$, and $A / K$ and $B / M$ are divisible groups.

Because $\widehat{R}$ is commutative $\operatorname{End}(A)=\operatorname{End}_{\widehat{R}}(A)$, and by $2.1 \operatorname{End}_{R}(A)=$ $\operatorname{End}_{\widehat{R}}(A)=\operatorname{End}_{R}(B)$.

However, $\operatorname{Hom}_{R}(B, A)=0$ as follows. Let $f \in \operatorname{Hom}_{R}(B, A)$. Because $M$ is $X$-divisible and $A$ is $X$-reduced, $f(M)=0$. Then $f(B)$, being a quotient of $B / M$, is a divisible subgroup of $A$. Inasmuch as $A$ is reduced, $f(B)=0$.

By using 2.13 and the modules given in 3.8 and 3.9 we can construct self-small mixed Corner $S[X]$-modules $A$ and $B$ such that $\operatorname{End}_{S[X]}(A) \cong \operatorname{End}_{S[X]}(B)=S[X]$, but $\operatorname{Hom}_{S[X]}(B, A)=0$. 
TOTALLY INDECOMPOSABLE MODULes. The module $A$ is totally indecomposable if $\operatorname{End}_{R}(A)$ has local classical ring of quotients. If $\operatorname{End}_{R}(A)$ is a commutative integral domain then $A$ is a totally indecomposable module. The (totally) indecomposable reduced torsion groups $\boldsymbol{A}$ are the primary cyclic groups. The torsion-free Abelian group $A$ of finite rank is totally indecomposable if and only if given a direct sum $B \oplus D$ and a nonzero integer $k$ such that $k A \subset B \oplus D \subset A$ then either $B=0$ or $D=0$.

The modules $A$ constructed in 3.11 show that these results do not extend to $R=$ $\mathbf{Z}[\boldsymbol{X}]$.

EXAMPLE 3.13.

1. There is a self-small totally indecomposable mixed Corner $S[X]$-module.

2. There is a totally indecomposable $X$-torsion Corner $S[X]$-module $A$ such that $X A \subset B \oplus D \subset A$ for some nonzero $S[X]$-submodules $B$ and $D$.

Proof: Let $R=S[X]$.

(1) Use 3.5 and the module $M$ given in 3.8 to construct a self-small mixed Corner module $A$ such that $M$ is the torsion submodule of $A$ and $\operatorname{End}(A)=R$. Then $A$ is totally indecomposable.

(2) Let $M$ be the module in 3.8. By 3.11 there is an $X$-torsion Corner module $A$ such that $M \subset A$ and $\operatorname{End}(A)=S[[X]]$.

Now by 2.6.2 there is a submodule $C=N+\sum_{m n} R \epsilon_{m n} \subset \bar{M}$ such that $\mathbf{Q} A=$ $\mathbf{Q} M \oplus \mathbf{Q} C$ and by $3.8 \mathbf{Q} M=\mathbf{Q} B \oplus \mathbf{Q} B^{\prime}$ where $B \cong R /(X)$. Then

$$
\mathbf{Q} A=\mathbf{Q} B \oplus \mathbf{Q} B^{\prime} \oplus \mathbf{Q} C
$$

as modules. An application of $X$ to (4) reveals that $X \mathbf{Q} A \cap \mathbf{Q} B=0$. Hence $X A \subset$ $B \oplus X A \subset A$, which completes the proof.

VANISHING TENSOR PRODUCTS. Let $R$ be a (commutative) ring and let $A$ be a module. Because tensor products commute with direct limits, if $T$ is a module such that $T_{0} \otimes_{R}$ $A=0$ for each finitely generated submodule $T_{0} \subset T$ then $T \otimes_{R} A=0$. The present examples show that the converse is not true. We require a Lemma.

Lemma 3.14. If $R$ is a localisation of $S[X]$ at a prime ideal $I$, if $X \in I$, and if $I \cap S=P$ then $R$ is a subring of $S_{P}[[X]]$ where $S_{p}$ is the localisation of $S$ at the prime ideal $P$. Moreover, $S_{P}[[X]] / R$ is a torsion-free $S$-module, and $S_{P}[[X]]$ is the completion of $R$ in the relativised topology on $R$.

Proof: Let $R$ denote the localisation of $S$ at the prime ideal $I$, suppose $X \in I$, and let $I \cap S=P$. Then given $q(X) \in S[X] \backslash I, q(0) \in S_{P}$ is a unit in $S_{P}$. Thus $q(X)$ is a unit of $S_{P}[[X]]$, and hence the universal property of localisations lifts the natural imbedding $S[X] \rightarrow S_{P}[[X]]$ to an imbedding $R \rightarrow S_{P}[[X]]$. Because $S[X] \subset R, S[[X]]$ is the completion of $R$. 
Moreover, assume there are $p(X) \in F S_{p}[[X]]$ and $s \in S$ such that $s p(X) \in R$. Because $R=S[X]_{I}$ there are $a(X) \in S[X]$ and $b(X) \in S[X] \backslash I$ such that $s p(X)=$ $a(X) b(X)^{-1}$. Then $p(X) b(X)=s^{-1} a(X) \in Q[X] \cap S_{P}[[X]]=S_{P}[X] \subset R$, where $Q$ is the field of quotients of $S$. Inasmuch as $b(X)$ is a unit of $R, p(X) \in R$ which completes the proof.

EXAmple 3.15. There is a local commutative Noetherian integral domain $R$ and a self-small Corner module $A$ such that

1. $R=\operatorname{End}(A)$

2. $T_{0} \otimes_{R} A \neq 0$ for each nonzero finitely generated right module $T_{0}$; but

3. There is a nonzero right module $T$ such that $T \otimes_{R} A=0$.

Proof: Let $p \in \mathbf{Z}$ be a prime, let $\mathbf{Z}_{p}$ denote the localisation of $\mathbf{Z}$ at $p$, and let $R$ be the localisation of $\mathbf{Z}_{p}[X]$ at the maximal ideal $(p, X)$. By $3.14 R$ is a pure subring of $\mathbf{Z}_{p}[[X]]$ and $\mathbf{Q}_{p}[[X]]$ is the completion of $R$ in the relativised $X$-adic topology on $R$. Then the $X$-torsion $Z_{p}[X]$-module $M$ given in 3.8 is also a module such that $\operatorname{ann}_{R}(M)=0$. (Let $S=Z_{p}$ in 3.8.) Furthermore, by 3.10 and 3.14

$$
\mathcal{O}(M)=\widehat{\mathcal{O}}(M) \bigcap \mathbf{Q} R=\mathbf{Z}_{p}[[X]] \bigcap \mathbf{Q} R=R .
$$

An application of 2.13 to $M$ shows that $M$ is the torsion submodule of a self-small mixed module $A$ such that $R=\operatorname{End}(A)$ and such that $A / M$ is a divisible group. Then $A$ satisfies 3.15.1.

Let $J \subset R$ be the unique maximal ideal of $R$. To prove that $A$ satisfies 3.15 .2 it is enough to show that $J A \neq A$. (See [10, Corollary 3.8(b)] or [2, Corollary 2.2].) By $3.8 M$ contains a direct summand isomorphic to the Corner module $R /(X) \cong \mathbf{Z}_{p}$, and because $(X) \subset J, J /(X) \neq R /(X)$. Thus $J M \neq M$, which by 3.1.1 implies $J A \neq A$. Thus $A$ satisfies 3.15.2.

Lastly, let $T$ be an $X$-divisible $p$-torsion module. (For example, choose $T$ to be the quotient field of $R / p R$.) Then $T \otimes_{R} M=0$ because $T$ is $X$-divisible and $M$ is $X$ torsion, while $T \otimes_{R} A / M=0$ because $T$ is $p$-torsion and $A / M$ is divisible. Therefore, $T \otimes_{R} A=0$, and the proof is complete.

It is natural to ask if $T \otimes_{R} A=0$ for each finitely generated module $T$ when $T_{0} \otimes_{R} A=0$ for each finitely presented module $T_{0}$.

EXAMPLE 3.16. There is a local commutative integral domain $R$ and a self-small Corner module $A$ such that

1. $R=\operatorname{End}(A)$;

2. $T_{0} \otimes_{R} A \neq 0$ for each nonzero finitely presented module $T_{0}$; but

3. $T \otimes_{R} A=0$ for some nonzero finitely generated module $T$. 
Proof: Let $p \in \mathbf{Z}$ be prime, let $\mathbf{Z}_{p}$ denote the localisation of $\mathbf{Z}$ at $p$, let $X_{1}, X_{2}, \ldots$ be countably many indeterminants over $Z_{p}$, and let $R$ denote the localisation of $Z\left[X_{1}, X_{2}, \ldots\right]$ at the maximal ideal $\left(p, X_{1}, X_{2}, \ldots\right)$. Then $R$ is a local commutative integral domain, and as in $3.14 R$ is a pure subring of the completion of $\mathbf{Z}_{p}\left[X_{1}, X_{2}, \ldots\right]$ in the $\left(X_{1}, X_{2}, \ldots\right)$-adic topology. Let $J$ denote the unique maximal ideal of $R$ and notice that $p \in J$.

We shall construct a module $M$ such that $J M=M$ but $I M \neq M$ for each finitely generated proper ideal $I \subset R$.

For each positive integer $k$ let $M_{k}$ be the $X_{k}$-divisible $X_{k}$-torsion $\mathbf{Z}_{p}\left[X_{k}\right]$-module given in 3.9 , and let

$$
M=\bigoplus_{k>0} M_{k} .
$$

Inasmuch as $M_{k}$ is a free $\mathbf{Z}_{p}$-module for each $k>0,3.9$, we have $p M \neq M$.

Now, $M_{k}$ is an $X_{k}$-torsion $Z_{p}\left[\left[X_{k}\right]\right]$-module. Furthermore, as in 3.14 one proves that the ring homomorphism $\mathbf{Z}_{p}\left[X_{1}, X_{2}, \ldots\right] \rightarrow \mathbf{Z}_{p}\left[\left[X_{k}\right]\right]$ such that $X_{k} \mapsto X_{k}$ and $X_{j} \mapsto 0$ for $j \neq k$ lifts to a ring homomorphism $R \rightarrow Z_{p}\left[\left[X_{k}\right]\right]$. Then $M_{k}$ is a torsion module such that $X_{j} M_{k}=0$ for each $j \neq k$.

3.17. Because $M_{k}=X_{k} M_{k}$ and because $X_{k} \in J$ we have $J M=M$. However, if $I \subset R$ is a finitely generated ideal then $I \subset I_{0}=\left(p, X_{1}, \ldots, X_{0}\right)$ for some integer $s$. Because $X_{j} M_{x+1}=0$ for each $j=1, \ldots, s$ and because $p M_{s+1} \neq M_{a+1}$ we have $I_{0} M_{a+1} \neq M_{a+1}$, and so $I M \subset I_{0} M \neq M$.

Now, because $R$ is pure subring of $S\left[\left[X_{1}, X_{2}, \ldots\right]\right]$ and because $S\left[\left[X_{1}, X_{2}, \ldots\right]\right]=$ $\widehat{\mathcal{O}}(M)$ we have $\mathcal{O}(R, M)=S\left[\left[X_{1}, X_{2}, \ldots\right]\right] \cap Q R=R$. Then by 2.13 there is a selfsmall mixed Corner group $A$ such that $M \subset A, R=\operatorname{End}(A)$, and $A / M$ is a divisible group.

Because $J M=M, 3.1 .1$ and 3.17 imply that $J A=A$. Next, let $I \subset R$ be a finitely generated ideal. By 3.17 there is a finitely generated ideal $I \subset I_{0} \subset R$ such that $R / I_{0}$ is bounded and $I_{0} M \neq M$. Then by 3.1.1 $I A \subset I_{0} A \neq A$.

Finally, because $I A \neq A$ for each proper finitely generated ideal $I \subset R,[10$, Corollary 3.8(a)] states that $T_{0} \otimes_{R} A \neq 0$ for each nonzero finitely presented module $T_{0}$. Thus 3.16 .2 is satisfied. The nonzero finitely generated module $T$ needed to satisfy 3.16 .3 is $T=R / J$.

If $A$ is a torsion-free group of finite rank and if $\operatorname{End}(A)$ is a (sub)commutative ring then $I A \neq A$ for each maximal right ideal $I \subset \operatorname{End}(A)$. If $\operatorname{End}(A)$ is a semi-prime ring then $I A=A$ for at most finitely many maximal ideals $I \subset \operatorname{End}(A),[4,11,12]$. The following example shows that this result is not true for self-small groups $A$.

EXAMpLE 3.18. There is a self-small Corner group $A$ such that 
1. End $(A)$ is a countable commutative Noetherian integral domain; and

2. $I A=A$ for each of the infinitely many maximal ideals $I \subset$ End $(A)$.

Proof: Given a prime $p \in \mathbf{Z}$ let $R_{p}$ denote the localisation of $\mathrm{Z}[X]$ at the maximal ideal $(p, X)$, and let

$$
R=\bigcap_{\text {primes }} \boldsymbol{R}_{\boldsymbol{p} \in \mathbf{Z}}
$$

Then $R$ is a countable ring and a maximal ideal of $R$ is generated by $\{p, X\}$ for some $p \in \mathbf{Z}$ is prime. Thus $R$ has infinitely many maximal ideals. Furthermore, 3.14 shows that we may view $R_{p}$ as a subring of $\mathbf{Z}_{p}[[x]\}$ for each prime $p \in Z$. Then $R$ is a subring of $\mathrm{Z}[[X]]$, so that the $X$-torsion $\mathrm{Z}[X]$-module $M$ given in 3.9 is also a module.

As in $3.15 R=\mathcal{O}(M)$, so by 2.13 there is a self-small Corner module $A$ such that $M \subset A, R=\operatorname{End}(A)$, and $A / M$ is a divisible group. Let $I=(p, X)$ be an ideal in $R$. Arguing as in 3.15 (with $X=J$ ) shows that $I A=X A+p A=A$. This completes the proof.

The Baer SPlitting Property. Let $c$ and $d$ be cardinal numbers, and consider a surjection $g: A^{(c)} \rightarrow A^{(d)}$ of groups. The group $A$ has the Baer splitting property if $g$ is a split surjection for each pair of cardinals $(c, d), A$ has the finite Baer splitting property if $g$ is a split surjection for each cardinal $c$ and integer $d$, and $A$ has the endlich Baer splitting property if $g$ is a split surjection for each pair of integers $(c, d)$. (See $[2,4,10]$.)

It is clear that the Baer splitting property implies the finite Baer splitting property implies the endlich Baer splitting property. The converses are not true for self-small groups.

EXAMPLE 3.19.

1. There is a self-small Corner group that has the finite Baer splitting property, but which does not have the Baer splitting property.

2. There is a self-small Corner group that has the endlich Baer splitting property, but which does not have the finite Baer splitting property.

Proof: (1) Let $A$ be the self-small Corner group constructed in 3.15. Because $T \otimes_{\operatorname{End}(A)} A=0$ for some nonzero $\operatorname{End}(A)$-module $T,[2$, Theorem 2.1] implies that $A$ does not have the Baer splitting property. However, because $T_{0} \otimes_{\operatorname{End}(A)} A \neq 0$ for each nonzero finitely generated $\operatorname{End}(A)$-module $T_{0},[2$, Corollary 2.2] shows that $A$ has the finite Baer splitting property.

(2) Let $A$ be the self-small Corner group constructed in 3.16. Because $T \otimes_{\operatorname{End}(A)}$ $A=0$ for some nonzero finitely generated $\operatorname{End}(A)$-module $T,[2$, Corollary 2.2] implies that $A$ does not have the finite Baer splitting property. However, because $T_{0} \otimes_{\operatorname{End}(\Lambda)}$ 
$A \neq 0$ for each nonzero finitely presented $\operatorname{End}(A)$-module $T_{0},[10$, Corollary 5.2$]$ shows that $A$ has the endlich Baer splitting property.

The above Example is in contrast to [10, Corollary 7.2] where it is shown that a torsion-free group of finite rank has the endlich Baer splitting property if and only if it has the finite Baer splitting property. It is interesting to note that $\operatorname{End}(A)$ is a local commutative Noetherian integral domain in 3.19.1, and that $\operatorname{End}(A)$ is a local commutative integral domain in 3.19.2.

Arnold and Lady [4] show that if $A$ is a torsion-free group of finite rank and if $\operatorname{End}(A)$ is a commutative ring then $A$ has the finite Baer splitting property, and [12, Lemma 3.1] shows that $A$ has the endlich Baer splitting property if $\operatorname{End}(A)$ is a local ring. Then by [10, Corollary 5.2$]$ the group constructed in 3.18 fits the following description.

EXAMPLE 3.20. There is a self-small Corner group $A$ such that $\operatorname{End}(A)$ is a local commutative Noetherian integral domain, but $A$ does not have the endlich Baer splitting property.

A Theorem of Azumaya's states that $A$ has the exchange property if $\operatorname{End}(A)$ is a local ring. Thus 3.19 and 3.20 show that

Proposition 3.21. The Exchange Property does not imply the (endlich) Baer splitting property.

\section{REFERENCES}

[1] F.W. Anderson and K.R. Fuller, Rings and categories of modules, Graduate texts in Mathematics 13 (Springer-Verlag, Berlin, Heidelberg, New York, 1974).

[2] U. Albrecht, 'Faithful Abelian groups of infinite rank', Proc. Amer. Math. Soc. 103 (1988), 21-26.

[3] D.M Arnold, Finite rank torsion-free Abelian groups and rings, Lecture Notes in Mathematics 931 (Springer-Verlag, Berlin, Heidelberg, New York, 1982).

[4] D.M. Arnold and L. Lady, 'Endomorphism rings and direct sums of torsion-free Abelian groups', Trans. Amer. Math. Soc. 211 (1975), 225-237.

[5] D.M. Arnold and C.E. Murley, 'Abelian groups $A$ such that Hom $(A, \cdot)$ preserves direct sums of copies of $A^{\prime}$, Pacific J. Math. 56 (1975), 7-20.

[6] A.L.S. Corner, 'Every countable torsion-free ring is an endomorphism ring', Proc. London Math. Soc. 13 (1963), 23-33.

[7] A.L.S. Corner, 'Endomorphism rings of torsion-free Abelian groups', in Proceedings of the International Conference on the Theory of Groups, Australian Nat. Univ., Canberra, August 1965, (L.C. Kovacs and B.H. Neumann, Editors) (Gordan and Breach Sci. Publishers, Inc., 1963), pp. 59-69.

[8] A.L.S. Corner and R. Gobel, 'Prescribing endomorphism algebras', Proc. London Math. Soc. 50 (1985), 447-494. 
[9] M. Dugas and T.G. Faticoni, 'Cotorsion-free Abelian groups cotorsion as modules over their endomorphism rings', in Abelian groups, Proceedings of the 1991 Curacao conference, (L. Fuchs and R. Gobel, Editors), Lecture Notes in Pure and Applied Mathematics 146 (Marcel Dekker, Inc., New York, Basel, Hong Kong, 1993), pp. 111-128.

[10] T.G. Faticoni, 'The endlich Baer splitting property', Pacific J. Math. 157 (1993), 225-240.

[11] T.G. Faticoni, 'Gabriel filters on the endomorphism ring of a torsion-free Abelian group', Comm. Algebra 18 (1990), 2841-2883.

[12] T.G. Faticoni, 'On the lattice of right ideals of the endomorphism ring of an Abelian group', Bull. Austral. Math. Soc. 38 (1988), 273-291.

[13] T.G. Faticoni, 'Each countable reduced torsion-free commutative ring is a pure subring of an E-ring', Comm. Algebra 15 (1987), 2545-2564.

[14] T.G. Faticoni and H. Pat Goeters, 'Examples of torsion-free groups flat as modules over their endomorphism rings', Comm. Algebra 19 (1991), 1-28.

[15] L. Fuchs, Infinite Abelian Groups I, II (Academic Press, New York-London, 1969, 1970).

[16] J. Reid, 'Abelian groups finitely generated over their endomorphism rings', in Lecture Notes in Mathematics 874 (Springer-Verlag, Berlin, Heidelberg, New York, 1981), pp. 41-52.

Department of Mathematics

Fordham University

Bronx

New York 10458

United States of America 